\title{
Assessment of Dietary Intakes and Food habits in Female Adolescents with Eating Disorders
}

\author{
Roshanak Roustaee ${ }^{1}$, Anahita Houshiar $\operatorname{Rad}^{2}$, Esra Tajik ${ }^{2}$, Majid Hajifaraji ${ }^{2} *$ \\ 1- PhD Student in Food and Nutrition Policy, National nutrition and Food Technology Research Institute, Faculty of Nutrition and Food Sciences, Shahid \\ Beheshti Medical University, Tehran, Iran \\ 2- National Nutrition and Food Technology Research Institute, Faculty of Nutrition Sciences and Food Technology, Shahid Beheshti University of \\ Medical Sciences, Tehran, Iran.
}

\section{A B S T R A C T}

Background and Objectives: Eating disorders are psychological syndromes which characterized by abnormal or disturbed food habits resulted from intense fear of obesity and may include negative effects on people physical or mental health. This cross sectional study was carried out to assess dietary intakes and food habits in female adolescents with eating disorders in Tehran, Iran.

Materials and Methods: Totally, 231 female adolescent with eating disorders were selected from a total number of 2766 students from five districts in Tehran using multistage clustered sampling in a 2-phase procedure. All affected students and 284 non-affected students filled demographic and food habit questionnaires (modified version) for adolescents and then were interviewed for the 70-item non-quantitative food frequency by expert nutritionists.

Results: Study of dietary patterns in adolescents showed that the most common meal pattern (30\%) in nonaffected group included three main meals and two snacks. The highest frequent patterns in partial syndrome group $(33.5 \%)$ and bulimia nervosa $(28.1 \%)$ included two main meals daily. The missing dinner in partial syndrome and bulimia nervosa groups were significantly higher than that in non-affected group $(\mathrm{P}<0.05)$. The highest frequency of drinking water (4-6 cups/day) was seen in partial syndrome group (41\%). This included 3-2 cups/day (39\% and 42.3\%) in bulimia nervosa and non-affected groups, respectively. A significant difference was seen between these groups $(\mathrm{P}=0.037)$. Consumption of all cereals and vegetables in the affected group was significantly lower than that in non-affected group $(\mathrm{P}<0.05)$. Study of lipid intake status in the participants showed that the frequency of low-fat dairy products in affected groups $(25 \%$ and $35.8 \%$ in partial syndrome and bulimia nervosa, respectively) was significantly higher than that in non-affected group $(12.7 \%)$.

Conclusions: Study demonstrated that missing dinner and drinking higher volumes of water with decreased cereal and low-fat dairies intakes were the major techniques used by the adolescents to reduce the calorie intake. Despite of these differences, the overall food habits in the affected group do not seem different significantly from those in non-affected groups.

Keywords: Dietary intake, Food habits, Eating disorders, Adolescent, Female

\section{Introduction}

Eating disorders (including anorexia nervosa, bulimia nervosa and partial syndrome) include psychological syndromes that are characterized by concerning of body weight and shape as well as a wide range of abnormal eating and include - from excessive diets or regimen to a full syndrome (1). Based on the DSM-IV criteria, anorexia nervosa is defined by refusal to maintain body weight at or 
above a minimally normal weight for age and height, intense fear of gaining weight or becoming fat, disturbance in way that the person's body weight or shape is experienced, and amenorrhea in postmenarcheal females; means, the absence of at least three consecutive menstrual cycles. Bulimia nervosa is defined by recurrent episodes of binge eating characterized by eating in a discrete period of time (e.g. within any 2-h period), a quantity of food that is definitely larger than most people would eat during a similar period of time and under similar circumstances with a sense of lack of control over eating during the episode, in addition to recurrent inappropriate compensatory behavior to prevent weight gain such as self-induced vomiting, misuse of laxatives, diuretics, enemas and other medications, fasting and excessive exercises. The binge eating and inappropriate compensatory behavior occur at least twice a week for three months. EDNOS or partial syndrome disorders that do not meet the criteria for full syndromes mentioned above. Examples include all criteria of anorexia nervosa are met except that the patients have regular menses or the patients' current weight is in the normal range despite a significant weight loss. Furthermore, all the criteria of bulimia nervosa are met in female patients except that the binge eating and inappropriate compensatory mechanisms occur less than twice a week or for less than three months or the patient has normal body weight and uses inappropriate compensatory behaviors regularly after eating small quantities of foods (2). Researches reveal that a relatively large proportion of female adolescents face eating disorders. Adolescents, especially females, often pay particular attentions to their body weight and shape and deal with stresses associated with critical and challenging puberty. They may show wrong eating attitudes, which may result in eating behavior problems (3-6). In Iran, limited studies have assessed the frequency of eating disorders. In 2000, lifetime frequencies of $0.9 \%$ for $\mathrm{AN}, 3.2 \%$ for $\mathrm{BN}$ and $6.6 \%$ for partial syndrome were reported for the female teenagers (7). In another study on female students in 2009 , frequencies of bulimia nervosa and partial syndrome included 2.1 and $6.5 \%$ respectively while no cases of anorexia were reported (8). In a study in 2011, eating disorders were demonstrated in more than $13 \%$ of female university students in Yazd, Iran
(9). In a study on occurrence of eating disorders in female university in Tehran, $1.8 \%$ suffered from anorexia nervosa and $7.8 \%$ from bulimia nervosa (10). Examples of disordered eating behaviors include energy constraints for weight loss, avoiding certain foods especially high-energy foods as fattening foods, eating a limited range of foods, limiting dietary fat intake, reducing food related flexibility and filling with low-energy foods. Other misbehaviors include excessively using artificial sweeteners, excessively using fruits and vegetables, using non-nutritious drinks such as water and diet drinks to suppress appetite and reduce eating desire and excessively consuming caffeine. Moreover, abnormal timing of eating, excessive interfering in food preparation, collecting recipes and menus, excessively using seasonings, overestimating food and confusion about the amount of food are other examples of abnormal eating behaviors. These unhealthy habits may develop disease progression. Furthermore, poor food quality and undesirable energy balance can lead to development of disorders in unfavorable cycles (11, 12). The high occurrence and harmful consequence of eating disorders cause health problems in adolescents. For example, studies have reported that teenagers with partial eating disorder syndrome complain of physical problems such as chronic constipation, indigestion, nausea, vomiting, abdominal pain, fatigue, headache, hypotension and irregular heartbeat; with the most common complaints linked to digestive system. In addition, growth decline and menstrual delay are reported in children and teenagers without severe weight loss and calcium, iron, thiamine, zinc, copper, magnesium, riboflavin, folic acid and vitamins $\mathrm{C}, \mathrm{A}, \mathrm{E}, \mathrm{D}, \mathrm{B} 6$ and $\mathrm{B} 12$ deficiencies (3, 13-15). Therefore, eating disorders can alter health situations with dietary pattern changes and consequently inadequate nutrients, which can result in nutritional disorders. Based on the age of individuals with eating disorders (adolescence and early adulthood) and its association with beginning of social activities and individual efficiencies, this problem matters much further. Since no studies have been carried out on food intake in people with eating disorders in Iran, this study was carried out to assess dietary intakes and food habits of female teens with eating disorders in Tehran, Iran. 


\section{Materials and Methods}

In the current descriptive cross-sectional study, 515 samples were selected within 2766 students who were investigated for the eating disorder occurrence. The sample size was calculated based on the frequency of eating disorders in study of Nobakht et al. (7). Students from public and non-profit schools were selected in five districts of Tehran (districts 1, 16, 8, 5 and 6 respectively in North, South, East, West and Center of Tehran) using multistage sampling method and according to proportion- of students in each district. In this study, demographic questionnaires included questions on the household size, parents' marital status, occupation and education as well as questions on date and order of student birth and age of menarche. The EAT-26 (Eating Attitude Test-26) questionnaire, validated by Nobakht et al., was used to screen and identify suspected people of eating disorders. Each question was -scored based on the Likert scale ranging from always (Score 3) to never (Score 0). Therefore, EAT-26 scores can range 0-78. Score 20 and above show - the risk of eating disorders (7). Furthermore, eating disorders were diagnosed using a structured clinical interview form. This was based on the Diagnostic and Statistical Manual of Mental Disorders-IV (DSM-IV), assessed in a study by Nobakht et al. for its reliability and validity (7). To study the usual dietary intake of the participants, a food frequency questionnaire with 168 items was used. Validity and reliability of the questionnaire were previously assessed by Mirmiran et al. (16). However, due to the time limit for data collection in schools, specific characteristics of the participants, published studies on teenagers and their various food intakes, items in the questionnaire decreased to 70 items of non-quantitative. The reliability of the new questionnaire was re-assessed by $20 \%$ of the population, based on the Cronbach's alpha coefficient $(\mathrm{r}=0.68)$. Moreover, food habits were assessed using valid food habit questionnaire (17). The section of food habits in this questionnaire included questions on eating main meals and snacks (always, more often, sometimes and never) which reports the frequency of consumption for each meal and snack. The major components of each main meal can be reported in the five major food groups and the most popular snacks and drinks. The questionnaire was translated into Farsi and non-compliance items with Iranian food culture were removed. Reliability of the new questionnaire was re-assessed by $20 \%$ of the population, which was relatively reliable based on the Cronbach's alpha coefficient $(r=0.72)$.

Data collection process: First, all students $(n=2766)$ completed demographic and eating disorder screening questionnaires (EAT-26). Students who scored over 20 points in the screening questionnaire $(\mathrm{n}=575)$ who were at risk of eating disorders completed eating disorders diagnostic questionnaire in the second stage. All participants were interviewed shortly for confirmation of the accurate conceptions and responses. Zero case of anorexia nervosa, 59 cases of bulimia nervosa and 178 cases of eating disorders not otherwise specified (EDNOS) or partial syndrome, totally, 237 person were diagnosed with eating disorders. To assess possible associations between eating disorders and food habits, 293 students (16\%) with a screening score below 15 were selected randomly and completed diagnostic questionnaires to ensure lack of eating disorders. These participated in the study as the non-affected group. All affected $(\mathrm{n}=$ $231)$ and non-affected $(n=284)$ participants filled the food habits questionnaire and interviewed by expert nutritionists for food frequency. The participants' weight and height were assessed using standard methods. Weight and height of the selected samples were recorded using standard methods by a trained expert. Weights were recorded using a portable digital scale (Seca,Germany) with an accuracy of $10 \mathrm{~g}$ and heights using non-elastic tape measures with $0.1 \mathrm{~cm}$ accuracy.

Data analysis: Statistical analysis was carried out using SPSS Software v.16 (IBM Analytics, USA). Normality of data was checked using KolmogorovSmirnov test. Chi-square test was used for qualitative variables, Kruskal-Wallis test for comparing nonnormal variables and One-way ANOVA for comparing the mean of quantitative variables between the groups. 


\section{Results}

Demographic characteristics showed that the mean age and age of menarche in the students included $15.75 \pm 0.9$ and $12.7 \pm 1.16$ years, respectively. Nearly half of the students lived in 4-member families; $44 \%$ of them were the first child of the family. The majority of mothers (80\%) were housewives and fathers mostly (45.4\%) were independent workers. Educational levels in $24 \%$ of mothers and $41 \%$ of fathers were above diploma. Mean weight, height and BMI included $60.3 \pm 11.5(\mathrm{~kg}), 160.8 \pm 6.7(\mathrm{~cm}), 23.3$ $\pm 4.2\left(\mathrm{~kg} / \mathrm{m}^{2}\right)$ in affected and $60.7 \pm 12.4(\mathrm{~kg}), 160.9$ $\pm 5.5(\mathrm{~cm})$ and $23.4 \pm 4.6\left(\mathrm{~kg} / \mathrm{m}^{2}\right)$ in non-affected groups, respectively. Differences between the groups were not statistically significant. Study on dietary patterns in non-affected group showed that the most common pattern $(30 \%)$ included three main meals and two snacks. The most frequent pattern in partial syndrome group (33.5\%) and bulimia nervosa (28.1\%) included two main meals daily. Differences between breakfast and lunch consumptions were not significant in affected and non-affected groups. The most popular breakfast content in affected (39\%) and non-affected $(41.5 \%)$ groups included bread and cheese with tea or milk. The most frequent lunch in affected group included a combination of cereals, meats and vegetables (18.8\%) and in non-affected group cereal, meats and dairy products $(19.5 \%)$.

The missing dinner (never consumed) in affected groups was higher than that in non-affected group, while dinner constant consumption was higher in nonaffected group (Table 1). Differences between groups were significant based on Kruskal-Wallis test
$(\mathrm{P}<0.001)$. The highest frequency of dinner content in affected and non-affected groups (13 and 18.1\%, respectively) included a combination of cereals, meats and vegetables. Nibbling (unplanned and repetitious eating between meals/snacks with no loss of control) was seen in $80 \%$ of patients with bulimia nervosa, $69 \%$ of patients with partial syndrome and $49.8 \%$ of non-affected group. Differences between groups were statistically significant $(\mathrm{P}<0.001)$ based on the chisquare test. Study on drinking patterns demonstrated that the highest frequency of drinking per day was linked to water consumption in affected and nonaffected groups $(36.5 \%$ in bulimia nervosa, $40 \%$ in partial syndrome and $35.1 \%$ in non-affected groups); followed by coffee or instant coffee $(24.2 \%$ in bulimia nervosa $24 \%$ in partial syndrome and $30 \%$ in non-affected groups). The highest frequency of drinking water (4-6 cups/day) was observed in partial syndrome group (41\%). This included 3-2 cups/day in bulimia nervosa and non-affected groups (39\% and $42.3 \%$, respectively). There was a significant difference between groups ( $\mathrm{P}=0.037)$. Totally, $65 \%$ of the students were tea drinkers with the frequency of 2-3 cups/day (42 and 39\% in partial syndrome and bulimia nervosa, respectively). This included 1 cup/day (42\%) in non-affected group. Differences in tea intake were not statistically significant between the groups $(\mathrm{P}=0.137)$. Moreover, frequency of drinking tea without sugar included $18.4 \%$ in nonaffected and $32.5 \%$ in other groups with significant differences $(\mathrm{P}=0.001)$. Frequency of cereal and vegetable consumptions per day in affected group was significantly lower than that in non-affected group $(\mathrm{P}<0.05)$ (Table 2).

Table 1. Frequency of dinner consumption by the students in various groups

\begin{tabular}{lcccc}
\hline \multicolumn{1}{c}{ Study group } & $\begin{array}{c}\text { Affected No. (\%) } \\
\text { Bulimia nervosa Partial syndrome }\end{array}$ & Non-affected No. (\%) & Total No. (\%) \\
Dinner consumption & & & \\
\hline Always & $12(6.7)$ & $35(19.7)$ & $131(73.6)$ & $178(100)$ \\
Most of the time & $19(13.8)$ & $44(31.9)$ & $75(54.3)$ & $138(100)$ \\
Sometimes & $20(12.6)$ & $70(44)$ & $69(43.4)$ & $159(100)$ \\
Never & $6(15)$ & $25(62.5)$ & $9(22.5)$ & $40(100)$ \\
Total & $57(11)$ & $174(33.8)$ & $284(55.2)$ & $515(100)$ \\
\hline
\end{tabular}


Table 2. Average frequency of intake per day for food groups and food items in various groups

\begin{tabular}{|c|c|c|c|c|}
\hline \multirow[t]{2}{*}{ Study group } & \multicolumn{2}{|c|}{ Affected } & \multirow[t]{3}{*}{ Non-affected } & \multirow[t]{3}{*}{ Total } \\
\hline & Bulimia nervosa & Partial syndrome & & \\
\hline Food item & & & & \\
\hline Bread and cereals* & 2.200 .70 & $2.20 \pm 0.82$ & 2.500 .70 & 2.380 .76 \\
\hline Bread $^{*} \dagger$ & $1.10 \pm 0.52$ & $1.12 \pm 0.58$ & $1.26 \pm 0.57$ & $1.20 \pm 0.57$ \\
\hline Rice* & $0.91 \pm 0.45$ & $0.87 \pm 0.45$ & $1.07 \pm 0.33$ & $0.98 \pm 0.57$ \\
\hline Other cereals & $0.21 \pm 0.19$ & $0.20 \pm 0.45$ & $0.19 \pm 0.19$ & $0.19 \pm 0.20$ \\
\hline Vegetables*** & $8.70 \pm 0.20$ & $8.50 \pm 2.10$ & $9.20 \pm 2.10$ & $8.90 \pm 2.10$ \\
\hline Leafy vegetables & $0.14 \pm 0.10$ & $0.14 \pm 0.10$ & $0.14 \pm 0.08$ & $0.14 \pm 0.90$ \\
\hline Zucchini/eggplant $\dagger$ & $0.05 \pm 0.06$ & $0.08 \pm 0.15$ & $0.06 \pm 0.07$ & $0.06 \pm 0.07$ \\
\hline Starchy vegetables & $0.22 \pm 0.21$ & $0.20 \pm 0.19$ & $0.23 \pm 0.20$ & $0.22 \pm 0.19$ \\
\hline Raw vegetables** & $2.32 \pm 1.86$ & $2.47 \pm 2.10$ & $1.90 \pm 1.30$ & $2.10 \pm 1.70$ \\
\hline Fruits & $1.65 \pm 0.91$ & $1.80 \pm 1.24$ & $1.70 \pm 1.00$ & $1.80 \pm 1.10$ \\
\hline Apple ${ }^{* * *} \dagger$ & $0.54 \pm 0.54$ & $0.57 \pm 0.61$ & $0.45 \pm 0.43$ & $0.50 \pm 0.51$ \\
\hline Banana & $0.20 \pm 0.26$ & $0.25 \pm 0.32$ & $0.30 \pm 0.29$ & $0.27 \pm 0.30$ \\
\hline Citrus & $0.78 \pm 0.65$ & $0.81 \pm 0.70$ & $0.84 \pm 0.65$ & $0.82 \pm 0.66$ \\
\hline Other fruits & $0.13 \pm 0.14$ & $0.17 \pm 0.23$ & $0.16 \pm 0.18$ & $0.16 \pm 0.20$ \\
\hline Dried fruits & $0.27 \pm 0.37$ & $0.36 \pm 0.38$ & $0.29 \pm 0.40$ & $0.31 \pm 0.39$ \\
\hline Nuts & $0.68 \pm 0.60$ & $0.66 \pm 0.70$ & $0.73 \pm 0.71$ & $0.73 \pm 0.71$ \\
\hline Meats & $1.12 \pm 0.42$ & $1.17 \pm 0.44$ & $1.20 \pm 0.38$ & $1.20 \pm 0.41$ \\
\hline Red meats & $0.52 \pm 0.30$ & $0.55 \pm 0.27$ & $0.56 \pm 0.26$ & $0.56 \pm 0.27$ \\
\hline White meats & $0.33 \pm 0.22$ & $0.29 \pm 0.23$ & $0.38 \pm 0.20$ & $0.38 \pm 0.21$ \\
\hline Egg & $0.19 \pm 0.16$ & $0.16 \pm 0.17$ & $0.18 \pm 0.16$ & $0.17 \pm 0.16$ \\
\hline Other meat products & $0.07 \pm 0.09$ & $0.06 \pm 0.08$ & $0.08 \pm 0.08$ & $0.07 \pm 0.08$ \\
\hline Beans & $0.83 \pm 0.56$ & $0.81 \pm 0.57$ & $0.88 \pm 0.56$ & $0.85 \pm 0.56$ \\
\hline Milk and dairy products & $2.50 \pm 1.60$ & $2.60 \pm 1.50$ & $2.70 \pm 1.40$ & $2.60 \pm 1.50$ \\
\hline Oil and fat & $0.84 \pm 0.67$ & $0.88 \pm 0.77$ & $1.01 \pm 0.75$ & $0.95 \pm 0.75$ \\
\hline Olive and olive oil & $0.33 \pm 0.57$ & $0.33 \pm 0.54$ & $0.27 \pm 0.41$ & $0.30 \pm 0.48$ \\
\hline Other oils* & $0.51 \pm 0.53$ & $0.55 \pm 0.54$ & $0.74 \pm 0.59$ & $0.65 \pm 0.57$ \\
\hline Fast foods & $0.48 \pm 0.43$ & $0.49 \pm 0.46$ & $0.49 \pm 0.38$ & $0.49 \pm 0.42$ \\
\hline Salty junk foods & $0.62 \pm 0.57$ & $0.58 \pm 0.50$ & $0.52 \pm 0.44$ & $0.55 \pm 0.47$ \\
\hline Sour junk foods* & $0.47 \pm 0.63$ & $0.40 \pm 0.42$ & $0.30 \pm 0.41$ & $0.35 \pm 0.44$ \\
\hline Sweet junk foods & $0.97 \pm 1.80$ & $0.85 \pm 1.14$ & $0.82 \pm 0.83$ & $0.85 \pm 1.08$ \\
\hline Pastry products & $0.62 \pm 0.46$ & $0.74 \pm 0.58$ & $0.74 \pm 0.53$ & $0.73 \pm 0.54$ \\
\hline
\end{tabular}

*Significant differences between affected and non-affected groups $(\mathrm{P}<0.001)$

$* \dagger$ Significant differences between affected and non-affected groups $(\mathrm{P}<0.05)$

**Significant differences between partial syndrome and non-affected groups $(\mathrm{P}<0.001)$

$* *+$ Significant differences between partial syndrome and non-affected groups $(\mathrm{P}<0.05)$

$\dagger$ Significant differences between affected and non-affected groups $(\mathrm{P}<0.05)$

$\ddagger$ Significant differences between bulimia nervosa and non-affected groups $(\mathrm{P}<0.05)$

One-way ANOVA analysis showed that the mean of bread consumption in bulimia nervosa group was significantly higher than that in partial syndrome and non-affected groups. However, the mean of rice consumption in partial syndrome group was significantly lower than that in other groups (Table 3). Totally, the average consumption of cereals in partial syndrome group was significantly lower than that in other groups. Study on lipid intake status in participants showed that the frequency of low-fat dairy products in affected groups (25 and $35.8 \%$ in partial syndrome and bulimia nervosa, respectively) was significantly higher than that in non-affected group (12.7\%). Frequency of Mayonnaise consumption in affected groups (81.5 and $79 \%$ in partial syndrome and bulimia nervosa, respectively) was significantly lower than that in non-affected group (93.3\%). 
Table 3. Average daily consumption of bread, rice and total cereals in students by groups (based on the available answers in questionnaires)

\begin{tabular}{lcr}
\hline Group & No. & Mean SD \\
\hline Bread (gr) & & \\
Partial syndrome & 167 & $89.3 \pm 106.2$ \\
Bulimia nervosa & 54 & $155.9 \pm 140.5^{\circ}$ \\
Non-affected & 283 & $96.9 \pm 106.6$ \\
Total & 504 & $100.7 \pm 112.1$ \\
Rice (gr) & & \\
Partial syndrome & 168 & $262.8 \pm 155.1^{\circ}$ \\
Bulimia nervosa & 56 & $301.8 \pm 193.5$ \\
Non-affected & 282 & $329.8 \pm 160.5$ \\
Total & 506 & $304.4 \pm 165.2$ \\
Bread and rice & & \\
Partial syndrome & 162 & $354.5 \pm 209.7^{\circ}$ \\
Bulimia nervosa & 53 & $457.6 \pm 246.2$ \\
Non-affected & 280 & $426.1 \pm 197.1$ \\
Total & 495 & $406.1 \pm 209.8$ \\
\hline
\end{tabular}

*Significant differences $(\mathrm{P}<0.05)$

\section{Disc ussion}

Study on food habits linked to frequent consumption of main meals showed that the frequency of missing one or two main meals in affected groups was significantly higher than that in non-affected group. No significant difference was seen in missing breakfast between the various groups. However, missing dinner in affected groups was significantly higher than that in non-affected group. Masheb et al. showed that women with bulimia nervosa had a higher rate of missing main meals especially lunch, compared to women in control group. Furthermore, the bulimia nervosa patients tended to have nibbling more than others; similar to the current study (18). In contrast, Gonidakis et al. demonstrated that the missing dinner was contributed to higher risk of eating disorders in nutrition students (19). Matheson et al. showed that further possibilities existed for the patients with bulimia nervosa or partial syndrome to deny lunches and midnight snacks (20). Patients seem to miss the main meals to reduce calorie intake for a better weight control. This was clearly seen in the current study for missing dinner. Elimination of dinner is frequently recommended as a method for the successful weight loss in Iran. Regarding the quantities of consumed liquids, the present study demonstrated that patients with eating disorders drank more water and tea than non-affected group did. Abraham et al. investigated that liquid intakes in eating disorders were significantly high (21). These findings were similar to those by Forboush and Mayer $(22,23)$. Patients seemed to use excessive quantities of liquids, especially energy-free drinks to suppress their appetite as a compensatory method in weight control. Generally, findings on the intake of liquids in patients with eating disorders are varied (24).. Results of frequent consumption of foods in the present study showed that consumption of cereals in affected group was slightly lower than that in non-affected group. Although this difference was statistically significant, it could be denied. The average consumption of cereals in partial syndrome group was significantly lower than that in other groups. A high percentage of the students in affected groups reported consumption of low-fat foods. In a study by Jauregui et al., patients with anorexia nervosa had lower consumption of bread and cereals, meat, sweet foods, and oily and fried foods. However, frequency of vegetable consumption was significantly high (25). Misra et al. found that people with anorexia received less calories from fats and more calories from carbohydrates and proteins, compared to control group (15). In the current study, however, a few limitations were reported, including several referrals of the research teams to schools for filling the questionnaires. This resulted in decreased cooperation of the school administrators and accuracy of the student answers. In addition, validity of food habit questionnaire in Iranian female and validity of new modified FFQ were not assessed. In general, patients with partial syndrome seemed to try reducing intakes of carbohydrates and fats to limit the energy intake for the loss of weight. In the present study, intake of 
breads in group with bulimia nervosa was significantly higher than that in other groups. Similarly, Fitzgibbon et al. investigated that the overweight people consumed more sugars and carbohydrates in overeating periods (26). However, in the current study, the cultural belief of fattening effects of rice and the avoidance of fattening foods might increase the consumption of breads in this group. Contrary to studies by Gendall et al. and Raymond et al $(27,28)$; in which, they found that bulimia nervosa patients in overeating periods received higher percentages of energy from fats, patients with bulimia nervosa in the present study received energy from low-fat foods. It might occur due to the knowledge of this group on the fattening effects of fats. Therefore, further detailed studies on the intake of macronutrients (especially fats) are necessary.

\section{Conclusion}

In general, study on food habits in the groups has shown that the missing dinner and high drink of liquids with decreased cereal and low-fat dairy intakes were the major techniques used by the students to reduce the calorie intake in the participants. Based on the results from the food intake frequency study, the overall patterns of food habits in affected groups do not seem significantly different from those in non-affected group.

\section{Financial disclosure}

The authors declared no financial interest.

\section{Funding/Support}

This research project was financially supported by National Nutrition and Food Technology Research Institute.

\section{References}

1. The American Dietetic Association: Nutrition intervention in the treatment of anorexia nervosa, bulimia nervosa, and other eating disorders. J Am Diet Assoc. 2006;106(12):2073.

2. Association AP. Diagnostic and statistical manual of mental disorders: DSM-IV-TR. American Psychiatric Pub; 2000.

3. Chamay-Weber C ,Narring F, Michaud P-A. Partial eating disorders among adolescents: A review. J Adolesc Health. 2005;37(5):416-26.

4. Yannakoulia M, Matalas A-L, Yiannakouris N, Papoutsakis C, Passos M, Klimis-Zacas D. Disordered eating attitudes: an emerging health problem among Mediterranean adolescents. Eat Weight Disord. 2004;9(2):126-33.

5. Jones JM, Bennett S, Olmsted MP, Lawson ML, Rodin $\mathrm{G}$.Disordered eating attitudes and behaviours in teenaged girls: a school-based study. CMAJ. 2001;165(5):547-52.

6. Falissard B. Eating disorders: interactions between human nutrition research and food behaviours. Trends in Food Science \& Technology. 2007;18(5):281-4.

7. Nobakht M, Dezhkam M. An epidemiological study of eating disorders in Iran. Int $\mathrm{J}$ Eat Disord. 2000;28(3):265-71.

8. Roustaee R, Hajifaraji M, Dezhkam M, Houshiar-rad A, Mehrabi Y, Zowghi T. Prevalence of eating disorders and some of the factors related to them among high school female students in the City of Tehran, 2010. Iranian J Nutr Sci Food Technol. 2013;8(1):135-44.

9. Nadjarzadeh A, Vaziri N, Naderi Z ,Daneshbodi H, Shamsi F, Lotfi MH. Assessment of the Eating Disorders in Female Students of Shahid Sadoughi University of Medical Sciences, Yazd, Iran, 2011. Journal of Community Health Research. 2012;1(2):84.

10. Roshandel A, Safavi M, Ghasemi I. Prevalence of Eating Disorders among Female Students of University (Tehran-Iran) 2012. life Science Journal.2012;9(4): 2822-8.

11. Steinglass JE, Glasofer DR, Walsh E, Guzman G, Peterson CB, Walsh BT, et al. Targeting habits in anorexia nervosa: a proof-of-concept randomized trial. Psychol Med. 2018;9:1-8.

12. Hart S, Marnane C, McMaster C, Thomas A. Development of the "Recovery from Eating Disorders for Life" Food Guide (REAL Food Guide) - a food pyramid for adults with an eating disorder. J Eat Disord. 2018;6:6.

13. Patrick L. Eating disorders: a review of the literature with emphasis on medical complications and clinical nutrition. Altern Med Rev. 2002;7(3):184-202.

14. Emans SJ. Pediatr Int. Pediatrics International. 2000;42(1):1-7.

15. Misra M, Tsai P, Anderson EJ, Hubbard JL, Gallagher $\mathrm{K}$, Soyka LA, et al. Nutrient intake in communitydwelling adolescent girls with anorexia nervosa and in healthy adolescents. Am J Clin Nutr. 2006;84(4):698706.

16. Mirmiran P, Esfahani FH, Mehrabi Y, Hedayati M, Azizi F. Reliability and relative validity of an FFQ for nutrients in the Tehran Lipid and Glucose Study. Public Health Nutr.2010;13(5):654-62.

17. Turconi G, Celsa M, Rezzani C, Biino G, Sartirana MA, Roggi C. Reliability of a dietary questionnaire on food habits, eating behaviour and nutritional knowledge of adolescents. Eur J Clin Nutr.2003;57:753. 
18. Masheb RM, Grilo CM, White MA. An examination of eating patterns in community women with bulimia nervosa and binge eating disorder. Int $\mathrm{J}$ Eat Disord. 2011;44(7):618-24.

19. Gonidakis F, Sigala A, Varsou E, Papadimitriou G. A study of eating attitudes and related factors in a sample of first-year female nutrition and dietetics students of Harokopion University in Athens, Greece. Eat Weight Disord. 2009;14(2-3):e121-e7.

20. Matheson BE, Tanofsky-Kraff M, Shafer-Berger S, Sedaka NM, Mooreville M, Reina SA, et al. Eating patterns in youth with and without loss of control eating. Int J Eat Disord. 2012;45(8):957-61.

21. Abraham SF, Hart S, Luscombe G, Russell J. Fluid intake, personality and behaviour in patients with eating disorders. Eat Weight Disord. 2006;11(1):e30-e4.

22. Mayer LE, Schebendach J, Bodell LP, Shingleton RM, Walsh BT. Eating behavior in anorexia nervosa: Before and after treatment. Int J Eat Disord. 2012;45(2):290-3.
23. Forbush KT, Hunt TK. Characterization of eating patterns among individuals with eating disorders: What is the state of the plate? Physiol Behav. 2014;134:92109.

24. Hart S, Abraham S, Luscombe G, Russell J. Fluid intake in patients with eating disorders. Int J Eat Disord. 2005;38(1):55-9.

25. Jauregui Lobera I, Bolanos Rios P. Choice of diet in patients with anorexia nervosa. Nutr Hosp. 2009;24(6):682-7

26. Fitzgibbon ML, Blackman LR. Binge eating disorder and bulimia nervosa: Differences in the quality and quantity of binge eating episodes. Int $\mathbf{J}$ Eat Disord. 2000;27(2):238-43.

27. Raymond NC, Neumeyer B, Warren CS, Lee SS , Peterson CB. Energy intake patterns in obese women with binge eating disorder. Obesity. 2003;11(7):869-79.

28. Gendall KA, Sullivan PE, Joyce PR, Carter FA, Bulik CM. The nutrient intake of women with bulimia nervosa. Int J Eat Disord. 1997;21(2):115-27. 